\title{
A Note on Hamiltonian Lie Group Actions and Massey Products
}

\author{
by \\ Zofia STĘPIEŃ and Aleksy TRALLE \\ Presented by Czestaw RYLL-NARDZEWSKI
}

\begin{abstract}
Summary. We show that the property of having only vanishing triple Massey products in equivariant cohomology is inherited by the set of fixed points of hamiltonian circle actions on closed symplectic manifolds. This result can be considered in a more general context of characterizing homotopic properties of Lie group actions. In particular it can be viewed as a partial answer to a question posed by Allday and Puppe about finding conditions ensuring the "formality" of $G$-actions.
\end{abstract}

1. Introduction. In this article we prove the following theorem.

THEOREM 1.1. Let $(M, \omega)$ be a closed symplectic manifold endowed with a hamiltonian circle action $S^{1} \times M \rightarrow M$. Let $F$ be any connected component of the fixed point set of this action. If there exists a non-vanishing triple Massey product in $H^{*}(F)$, then the same is valid for the equivariant cohomology $H_{S^{1}}^{*}(M)$.

Obviously, the above theorem can be rephrased as follows. If $(M, \omega)$ is a closed symplectic manifold endowed with a hamiltonian circle action $S^{1} \times M \rightarrow M$ such that all the triple Massey products in the equivariant cohomology $H_{S^{1}}^{*}(M)$ vanish, then any connected component $F$ of the fixed point set $M^{S^{1}}$ also has only vanishing Massey products in $H^{*}(F)$.

Thus, the vanishing of the triple Massey products is inherited by the connected components of the fixed point set. This result can be considered in a more general context of the cohomology theory of Lie transformation groups $[\mathrm{AP}]$. Allday and Puppe asked about possible characterizations of "formal" Lie group actions. Recall that a topological space $X$ is called formal

2000 Mathematics Subject Classification: 53C15, 55P62.

Key words and phrases: hamiltonian circle action, Massey product. 
if it has a minimal model $\mathcal{M}_{X}$ quasi-isomorphic to the cohomology algebra $H^{*}\left(\mathcal{M}_{X}\right)$ (the latter is isomorphic to the cohomology algebra $H^{*}(X)$ of $X$; we do not give any details here, referring to [AP, TO]). It is natural to ask if one can characterize (in terms of the invariants of the given $G$-action) the group actions with formal fixed point set $X^{G}$. Since the non-vanishing of Massey products is an obstruction to formality [TO] (see also references therein), Theorem 1.1 is a partial answer to the above question. It appears that the weaker property of vanishing of the triple Massey products is indeed inherited by the fixed points of hamiltonian circle actions.

On the other hand, this result can be viewed as a characterization of hamiltonian circle actions themselves, and in this respect develops the theory along the lines of Kirwan's result $[\mathrm{K}]$ that for a closed $(M, \omega)$ endowed with a hamiltonian action of a compact Lie group $G$ the Leray-Serre spectral sequence associated with the Borel fibration

$$
M \rightarrow E G \times_{G} M \rightarrow B G
$$

collapses at $E_{2}$. Note that characterizing hamiltonian and non-hamiltonian actions is an important question in symplectic topology (cf. [Au, G, JK, LO, $\mathrm{McD}, \mathrm{McDS}]$ ). For example, it is well known that in dimension 4, a circle action is hamiltonian if and only if it has fixed points $[\mathrm{Au}, \mathrm{McD}]$. This fact does not hold in higher dimensions, but the counterexample of McDuff is quite subtle. It is still not known if there exist non-hamiltonian circle actions with discrete set of fixed points [G].

Acknowledgments. We are grateful to Chris Allday who attracted our attention to the problem of "formality" of Lie group actions and shared with us his insights in the theory of group actions. Also, we express our thanks to Jarek Kędra and James Stasheff for useful remarks, and acknowledge the support of the Polish Committee for Scientific Research (KBN), grant 2P03A 03624 .

\section{Preliminaries and notation}

Massey products and the Cartan model. Here we recall briefly the notion of Massey products in the form suitable for our considerations. In this paper all algebras and cohomologies are considered over the reals. To get a more detailed exposition of this topic, one can consult [RT]. Note however, that we use the term "non-vanishing" or "non-zero" Massey product instead of "essential" Massey product in $[\mathrm{RT}]$. Let $(A, d)$ be a commutative differential graded algebra. The cohomology algebra of $(A, d)$ is denoted by $H^{*}(A)$. If $a \in A$ is a cocycle, we write $[a]$ for the corresponding cohomology class. For a homogeneous element $a \in A$ of degree $p$ we write $\bar{a}=(-1)^{p} a$. Assume that we are given a triple of cohomology classes $[a],[b],[c]$ such that $[a][b]=$ 
$[b][c]=0$. Consider $x \in A$ and $y \in A$ such that $d x=\bar{a} b$ and $d y=\bar{b} c$. One can check that $\bar{a} y+\bar{x} c$ is a cocycle and therefore determines a cohomology class $[\bar{a} y+\bar{x} c] \in H^{*}(A)$. Note that this class depends on the choice of $x$ and $y$. By definition the set of all cohomology classes $[\bar{a} y+\bar{x} c]$ is denoted by $\langle[a],[b],[c]\rangle$ and is called the triple Massey product.

Definition. We say that the triple Massey product $\langle[a],[b],[c]\rangle$ does not vanish if the set of all cohomology classes $[\bar{a} y+\bar{x} c]$ does not contain zero. In the opposite case we say that $\langle[a],[b],[c]\rangle$ vanishes.

One can easily describe the vanishing of the triple Massey product in the following way (see [RT, Prop. 1.5]). Let $\langle[a],[b],[c]\rangle$ be a triple Massey product in $H^{*}(A)$. Denote by $([a],[c])$ the ideal in $H^{*}(A)$ generated by $[a]$ and $[c]$. The product $\langle[a],[b],[c]\rangle$ does not vanish if and only if there exists a cohomology class $x \in\langle[a],[b],[c]\rangle$ such that $x \notin([a],[c])$.

We also need the following formulas (see [RT, Prop. 1.4]):

$$
\begin{aligned}
& \xi\left\langle a_{1}, a_{2}, a_{3}\right\rangle \subset\left\langle\xi a_{1}, a_{2}, a_{3}\right\rangle, \\
& \xi\left\langle a_{1}, a_{2}, a_{3}\right\rangle \subset\left\langle a_{1}, \xi a_{2}, a_{3}\right\rangle, \\
& \xi\left\langle a_{1}, a_{2}, a_{3}\right\rangle \subset\left\langle a_{1}, a_{2}, \xi a_{3}\right\rangle,
\end{aligned}
$$

which are valid for any $a_{1}, a_{2}, a_{3} \in H^{*}(A)$ and for any $\xi$ represented by a central element in $A$.

Let $f:(A, d) \rightarrow\left(A^{\prime}, d^{\prime}\right)$ be a morphism of differential graded algebras. Then

(see Prop. 1.3 in $[\mathrm{RT}]$ ).

Consider now the case of a $G$-manifold, i.e. a manifold endowed with a smooth action of a Lie group $G$. We use the equivariant cohomology of the $G$-manifold, i.e. the cohomology of the total space of the Borel fibration

$$
M \rightarrow E G \times_{G} M \rightarrow B G
$$

associated with the universal principal $G$-bundle $G \rightarrow E G \rightarrow B G$ over the classifying space $B G$ of the Lie group $G$. Thus, $H_{G}^{*}(M)=H^{*}\left(E G \times_{G} M\right)$ (see $[\mathrm{Au}, \mathrm{GS}]$ ).

In the proof of Theorem 1.1 we will calculate Massey products with respect to the Cartan model. Recall that with any $G$-manifold $M$ one can associate the following differential graded algebra. Let $\Omega^{*}(M)$ be the de Rham algebra of $M$, and $S\left(\mathfrak{g}^{*}\right)$ the symmetric algebra over the dual to the Lie algebra $\mathfrak{g}$ of $G$. Then $G$ acts on $\mathfrak{g}^{*}$ by the coadjoint representation and hence there is a natural $G$-action on the tensor product $\Omega^{*}(M) \otimes S\left(\mathfrak{g}^{*}\right)$. We consider the subalgebra $\Omega_{G}^{*}(M)=\left(\Omega^{*}(M) \otimes S\left(\mathfrak{g}^{*}\right)\right)^{G}$ of the fixed points of this action. The details of this construction can be found in [BV, GS, JK, McDS]. We use the fact that there is a natural differential $D: \Omega_{G}^{*}(M) \rightarrow$ 
$\Omega_{G}^{*}(M)$ and that

$$
H^{*}\left(\Omega_{G}^{*}(M), D\right) \cong H_{G}^{*}(M) .
$$

Hamiltonian G-actions. Finally, recall the definition of the hamiltonian action of a Lie group on a symplectic manifold [Au, GS, K, McDS]. Let $(M, \omega)$ be a symplectic manifold and let $G$ be a Lie group acting on $M$ by symplectomorphisms. A smooth map $\mu: M \rightarrow \mathfrak{g}^{*}$ is called a moment map of the given $G$-action if

(1) $\mu$ is $G$-equivariant with respect to the given $G$-action on $M$ and with respect to the coadjoint $G$-action on $\mathfrak{g}^{*}$;

(2) for any $m \in M, X \in \mathfrak{g}$ and $v \in T_{m} M$ the following equality holds:

$$
T_{m} \mu(v)(X)=\omega\left(v, \widetilde{X}_{m}\right)
$$

(where $\widetilde{X}$ denotes the fundamental vector field on $M$ generated by $X)$.

Definition 2.2. We call a symplectic $G$-action on $(M, \omega)$ hamiltonian if it has a moment map.

The moment map need not be unique. It is not difficult to check that any symplectic action of a compact Lie group on a closed symplectic manifold $M$ such that $H_{1}(M)=0$ is hamiltonian. On the other hand, in the case of manifolds with large fundamental groups, e.g. aspherical symplectic manifolds, the notion of hamiltonian action is related to interesting and non-trivial topological questions (cf. $[\mathrm{LO}, \mathrm{McD}]$ ).

3. Proof of Theorem 1.1. Consider the triple $(M, \omega, G)$. Recall that if the $G$-action is symplectic, then the fixed point set $M^{G}$ is a symplectic submanifold and it is a finite union of connected closed symplectic submanifolds:

$$
M^{G}=\bigcup_{i=1}^{p} F_{i} .
$$

Choose one of the connected components, say $F_{1}$, and denote it by $F$. We have a symplectic embedding

$$
i_{F}: F \rightarrow M .
$$

Consider $F$ as a symplectic $G$-manifold with a trivial action of $G$. In particular, $H_{G}^{*}(F)=H^{*}(F) \otimes S\left(\mathfrak{g}^{*}\right)^{G}$. In case $G=S^{1}$ we have

$$
H_{S^{1}}^{*}(F)=H^{*}(F) \otimes \mathbb{R}[h],
$$

where $\mathbb{R}[h]$ denotes the free polynomial algebra with one generator $h$ of degree 2. Passing to the Borel fibrations we obtain the following commutative 
diagram:

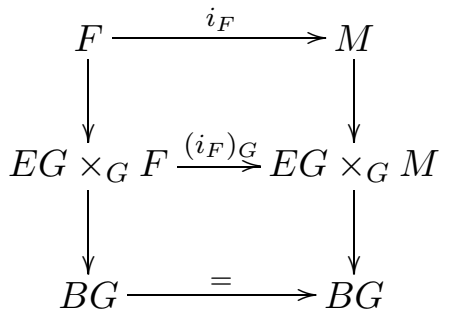

On the cohomology level we get the maps $\left(i_{F}\right)_{G}^{*}: H_{G}^{*}(M) \rightarrow H_{G}^{*}(F)$ and $i_{F}^{*}: H^{*}(M) \rightarrow H^{*}(F)$. Let $\nu$ denote the normal bundle of the symplectic embedding $i_{F}$. Since $G$ acts on this bundle fiberwise, one can define the equivariant normal bundle $\nu_{G}=E G \times_{G} \nu$. In this way we obtain the vector bundle

$$
E G \times_{G} \nu \rightarrow E_{G} \times_{G} F .
$$

In particular, the Euler class of (3.2) is called the equivariant Euler class of $\nu$. Throughout the paper, it is denoted by $\chi \in H_{G}^{*}(F)$.

The following facts can be found in [McDS, pp. 192-193] or in [JK].

Proposition 3.1. Let $G=S^{1}$ act on a closed symplectic manifold $(M, \omega)$ and let $F$ denote a connected component of $M^{G}$. Then:

(i) the normal bundle $\nu$ splits into the sum of complex line bundles

$$
\nu=\bigoplus_{j=1}^{m} L_{j}, \quad m=\operatorname{codim}_{M} F,
$$

invariant with respect to the $S^{1}$-action, where the circle group acts on each $L_{j}$ with weight $k_{j}$;

(ii) the equivariant Euler class has the form

$$
\chi=\prod_{j=1}^{m}\left(c_{1}\left(L_{j}\right)+k_{j} h\right),
$$

where $c_{1}\left(L_{j}\right)$ denotes the first Chern class of $L_{j}$.

Consider now the whole set $M^{G}=\bigcup_{k=1}^{p} F_{k}$ and apply the described constructions to each connected component $F_{k}$. In this way one gets the maps $\left(i_{F_{k}}\right)_{G}^{*}$ for all $k$, determining the map $\sum_{k}\left(i_{F_{k}}\right)_{G}^{*}: H_{G}^{*}(M) \rightarrow H_{G}^{*}\left(M^{G}\right)$. The following properties of these maps are valid for any hamiltonian $G$ action.

Proposition 3.2 ([Au, p. 139, JK, p. 5]). Assume that $G$ is a torus and acts on a closed symplectic manifold $(M, \omega)$ in a hamiltonian way. Then

(i) the $\operatorname{map} \sum_{F_{k}}\left(i_{F_{k}}\right)_{G}^{*}: H_{G}^{*}(M) \rightarrow H_{G}^{*}\left(M^{G}\right)$ is injective; 
(ii) there exists a linear map (the Gysin homomorphism) $\left(\left(i_{F}\right)_{G}\right)_{*}$ : $H_{G}^{*}(F) \rightarrow H_{G}^{*}(M)$ with the property

$$
\left(i_{F}\right)_{G}^{*}\left(\left(i_{F}\right)_{G}\right)_{*}(x)=\chi x \quad \text { for any } x \in H_{G}^{*}(F) ;
$$

(iii) $\chi$ is not a zero divisor in $H_{G}^{*}(F)$.

The Gysin map was introduced in $[\mathrm{Au}, \mathrm{JK}]$ in topological terms. We need an alternative way of describing it in the language of the Cartan complex. This was done in [GS, Chapter 10]. However, to use it, we need to prove (ii), since we do not know of any source where it is proved that the definitions of $\left(\left(i_{F}\right)_{G}\right)_{*}$ in $[\mathrm{Au}, \mathrm{J}]$ and $[\mathrm{GS}]$ coincide on the level of equivariant cohomology.

Let $\tau$ denote the equivariant Thom form of the equivariant normal bundle [GS]. Recall that $\tau \in \Omega_{G}^{*}(\nu)_{\mathrm{c}}$, where $\Omega_{G}^{*}(\nu)_{\mathrm{c}}$ denotes the Cartan complex of equivariant differential forms on the normal bundle $\nu$ with compact supports. If one identifies $\nu$ with the tubular neighbourhood of $F$ in $M$, one can extend $\tau$ onto $M$ by zero. Consider the natural projection $\pi: \nu \rightarrow F$. For any $\theta \in \Omega_{G}^{*}(F)$, define $i_{*} \theta$ as

$$
i_{*} \theta=\pi^{*} \theta \wedge \tau .
$$

Extending $i_{*} \theta$ onto the whole $M$ by zero, and passing to the equivariant cohomology, we get the Gysin map

$$
\left(\left(i_{F}\right)_{G}\right)_{*}: H_{G}^{*}(F) \rightarrow H_{G}^{*}(M) .
$$

Now the only thing we need is to verify (ii). Since in [GS, formula 10.12] it is shown that $\left(i_{F}\right)_{G}^{*} \tau=\chi$, the result follows by applying $\left(i_{F}\right)_{G}^{*}$ to both sides of (3.4).

The proof of Theorem 1.1 will now follow from the two lemmas below. Note that in both lemmas we keep the same notation and we assume that $G$ is a torus acting on $(M, \omega)$ in a hamiltonian way.

Lemma 3.1. Let $\langle u, v, w\rangle \subset H_{G}^{*}(F)$ be a triple Massey product. Then the following Massey product is defined in $H_{G}^{*}(F)$ :

$$
\langle\chi u, \chi v, \chi w\rangle \text {. }
$$

If the Massey product (3.5) does not contain zero, then the Massey product

$$
\left\langle\left(\left(i_{F}\right)_{G}\right)_{*} u,\left(\left(i_{F}\right)_{G}\right)_{*} v,\left(\left(i_{F}\right)_{G}\right)_{*} w\right\rangle \subset H_{G}^{*}(M)
$$

is defined and does not contain zero.

Proof. To avoid clumsy notation let us temporarily denote $\left(\left(i_{F}\right)_{G}\right)_{*}$ as $\left(i_{F}\right)_{*}$ and $\left(i_{F}\right)_{G}^{*}$ as $\left(i_{F}\right)^{*}$. Now we will need equivariant differential forms representing the equivariant cohomology. Let $U_{l}$ denote an equivariant tubular neighbourhood of $F_{l}$ (identified, as usual, with the equivariant normal bundle of the embedding), and let $[\tau]$ denote the equivariant Thom class represented by the equivariant differential form $\tau$ with compart support in $U_{l}$. Note that from the very definition of the Thom form and the Gysin map, the image 
of $\left(i_{F_{l}}\right)_{*}$ is contained in $U_{l}$ (see (3.4)). This obviously means that for any $x \in H_{G}^{*}\left(F_{l}\right)$ its image under the Gysin map in fact lies in the cohomology of $U_{l}$ with compact supports, and therefore its pullback by $i_{F_{k}}^{*}$ must be zero. Finally, we have obtained the equality

$$
i_{F_{k}}^{*}\left(i_{F_{l}}\right)_{*} x=0 \quad \text { for any } x \in H_{G}^{*}\left(F_{l}\right), l \neq k .
$$

Now we are ready to show that (3.5) is defined. By (3.7),

$$
\sum_{F_{k}} i_{F_{k}}^{*}\left(\left(i_{F}\right)_{*} u \cdot\left(i_{F}\right)_{*} v\right)=i_{F}^{*}\left(i_{F}\right)_{*} u \cdot i_{F}^{*}\left(i_{F}\right)_{*} v=\chi u \cdot \chi w=0,
$$

which obviously implies $\left(i_{F}\right)_{*} u\left(i_{F}\right)_{*} v=0$ because of Proposition 3.2. Obviuosly, the same could be written for $\left(i_{F}\right)_{*} v\left(i_{F}\right)_{*} w$. Note that the Gysin map is not multiplicative, but $i_{F}^{*}$ is, and this allows us to complete the proof. The following formulae show that (3.6) does not contain zero:

$$
i_{F}^{*}\left\langle\left(i_{F}\right)_{*} u,\left(i_{F}\right)_{*} v,\left(i_{F}\right)_{*} w\right\rangle \subset\left\langle i_{F}^{*}\left(i_{F}\right)_{*} u, i_{F}^{*}\left(i_{F}\right)_{*} v, i_{F}^{*}\left(i_{F}\right)_{*} w\right\rangle=\langle\chi u, \chi v, \chi w\rangle .
$$

Here we used (2.2) and Proposition 3.2.

Lemma 3.2. Assume $G=S^{1}$ and that $\langle u, v, w\rangle$ is a non-vanishing triple Massey product in $H^{*}(F) \subset H_{S^{1}}^{*}(F)=H^{*}(F) \otimes \mathbb{R}[h]$. Then

$$
\langle\chi u, \chi v, \chi w\rangle \neq 0 \text {. }
$$

Proof. According to Section 2, we need only prove that there exists $z \in\langle\chi u, \chi v, \chi w\rangle$ such that $z \notin(\chi u, \chi w)$. Use the equivariant cohomology $H_{S^{1}}^{*}(F)$ calculated with respect to the Cartan model. Take a non-trivial Massey triple product $\langle u, v, w\rangle$ considered as a non-trivial Massey product in the equivariant cohomology (one can easily check by straightforward calculation that $\langle u, v, w\rangle$ cannot become zero in the tensor product $H^{*}(F) \otimes \mathbb{R}[h]$ by writing the corresponding cocycles in the Cartan model). From Lemma 3.1, $\langle\chi u, \chi v, \chi w\rangle$ is defined. Since $\langle u, v, w\rangle \neq 0$, there exists $x \in\langle u, v, w\rangle$ such that $x \notin(u, w)$. Note that $\chi^{3} x \in\langle\chi u, \chi v, \chi w\rangle$ (by (2.1)). Assume that

$$
\langle\chi u, \chi v, \chi w\rangle=0 \text {. }
$$

This means that any $z \in\langle\chi u, \chi v, \chi w\rangle$ belongs to the ideal $(\chi u, \chi w) \subset$ $H_{S^{1}}^{*}(F)$. In particular, $\chi^{3} x \in(\chi u, \chi w)$. Hence

$$
\chi^{3} x=\chi u a+\chi w b, \quad a, b \in H_{S^{1}}^{*}(F) .
$$

Therefore

$$
\chi\left(\chi^{2} x-u a-w b\right)=0 .
$$

Recalling that $\chi$ is not a zero divisor (Proposition 3.2(iii)) one can write

$$
\chi^{2} x=u a+w b .
$$


Taking into consideration that $u, w \in H^{*}(F) \subset H_{S^{1}}^{*}(F)$ and the expression for the Euler class (3.3) one obtains

$\prod_{j=1}^{m}\left(c_{1}\left(L_{j}\right)+k_{j} h\right)^{2} x=u\left(a_{0}+a_{1} h+\cdots+a_{2 m} h^{2 m}\right)+w\left(b_{0}+b_{1} h+\cdots+b_{2 m} h^{2 m}\right)$.

Note that $h$ is a free generator, and $k_{1} \cdots k_{m} \neq 0$, because all $k_{j}$ are the weights of the normal representation of the circle. Using this and comparing the coefficients of $h^{2 m}$ on both sides of the latter equation yields $x \in(u, w)$, a contradiction. Finally, $z=\chi^{3} x$ is the required element.

Now we can complete the proof of Theorem 1.1. If $\langle u, v, w\rangle$ is a non-trivial triple Massey product in $H^{*}(F)$, then Lemma 3.2 implies that $\langle\chi u, \chi v, \chi w\rangle$ is a non-vanishing triple Massey product in $H_{S^{1}}^{*}(F)$. From Lemma 3.1 we get a non-trivial triple Massey product in $H_{G}^{*}(M)$ expressed by formula (3.6).

REMARK. In $[\mathrm{McD}]$ an example of a non-hamiltonian circle action on some closed 6-dimensional manifold was given. This action had a 2-dimensional submanifold of fixed points. Theorem 1.1 may yield another method of constructing non-hamiltonian circle actions with "big" sets of fixed points. Indeed, it would be sufficient to construct a closed symplectic $G$-manifold $M$ with vanishing triple Massey products but with $M^{G}$ having a non-vanishing one. However, this does not seem easy.

\section{References}

[AP] C. Allday and V. Puppe, Cohomology Theory of Transformation Groups, Cambridge Univ. Press, 1993.

[Au] M. Audin, The Topology of Torus Actions on Symplectic Manifolds, Birkhäuser, Basel, 1991.

[BV] M. Berline et M. Vergne, Zéros d'un champ de vecteurs et classes caractéristiques équivariantes, Duke Math. J. 50 (1983), 539-549.

[G] V. L. Ginzburg, Some remarks on symplectic actions of compact groups, Math. Z. 210 (1992), 625-640.

[GS] V. Guillemin and S. Sternberg, Supersymmetry and Equivariant de Rham Theory, Springer, Berlin, 1999.

[JK] L. Jeffrey and F. Kirwan, Applications of equivariant cohomology to symplectic geometry and moduli spaces, in: Austral. Math. Soc. Lect. Ser. 11, Cambridge Univ. Press, Cambridge, 1998, 1-19.

[K] F. Kirwan, Cohomology of Quotients in Symplectic and Algebraic Geometry, Princeton Univ. Press, 1984.

[LO] G. Lupton and J. Oprea, Cohomologically symplectic spaces. Toral actions and the Gottlieb group, Trans. Amer. Math. Soc. 347 (1995), 261-288.

[McD] D. McDuff, The moment map for circle actions on symplectic manifolds, J. Geom. Phys. 5 (1988), 149-160.

[McDS] D. McDuff and D. Salamon, Introduction to Symplectic Topology, Oxford Univ. Press, 1998. 
[RT] Y. Rudyak and A. Tralle, On Thom spaces, Massey products and non-formal symplectic manifolds, Internat. Math. Res. Notices 2000, no. 10, 495-513.

[TO] A. Tralle and J. Oprea, Symplectic Manifolds with no Kähler Structure, Springer, Berlin, 1997.

Zofia Stępień

Szczecin Technical University

Al. Piastów 38

70-323 Szczecin, Poland

E-mail: stepien@arcadia.tuniv.szczecin.pl
Aleksy Tralle

University of Warmia and Mazury

Żołnierska $14 \mathrm{~A}$

10-561 Olsztyn, Poland

E-mail: tralle@matman.uwm.edu.pl

Web: http://matman.uwm.edu.pl/ ${ }^{\sim}$ tralle

Received February 11, 2004;

received in final form March 8, 2004 